\title{
CARACTERIZACIÓN HIDROGEOLÓGICA DEL ACUÍFERO ALUVIAL NARANJITO, QUEPOS, PUNTARENAS
}

\author{
HYDROGEOLOGICAL CHARACTERIZATION OF THE NARANJITO ALLUVIAL \\ AQUIFER, QUEPOS, PUNTARENAS
}

\author{
Laura Castro \\ Compañía Nacional de Fuerza y Luz. Apartado postal: 10037-1000 San José \\ lcastro@cnfl.go.cr
}

(Recibido: 7/02/2007; aceptado: 22/06/2010)

\begin{abstract}
RESUMEN: El Acuífero Aluvial Naranjito es una de las tres fuentes de abastecimiento de agua potable de la comunidad de Quepos, por lo que se determina como principal objetivo describir la geología, los parámetros hidrogeológicos e hidrogeoquímicos de la cuenca baja del río Naranjo. El área de estudio se caracteriza por presentar rocas sedimentarias marinas y terrígenas del Terciario Inferior y del Cuaternario, que incluyen como principal unidad acuífera los depósitos aluviales, de velocidades de infiltración variables, alta transmisividad y alta conductividad hidráulica. A partir de la composición química de las muestras analizadas, se determinaron dos unidades de agua subterránea distintas, las cuales se clasifican dentro del tipo bicarbonatada cálcica y bicarbonatada clorurada sódica magnésica. Con ayuda de la exploración geofísica mediante la resistividad eléctrica, se obtiene información del acuífero, como la profundidad del nivel del agua, las variaciones laterales y en profundidad de los depósitos aluviales.
\end{abstract}

Palabras clave: Quepos, río Naranjo, acuífero Naranjito, hidrogeología, hidrogeoquímica.

\begin{abstract}
The Naranjito Alluvial Aquifer is one of the three public water supply sources of the community of Quepos, so it is determined as main objective to describe the geological, hydrogeological and hydrochemical features of the Naranjo River lower basin. The geology is characterized by Early Tertiary and Quaternary marine and terrigenous sedimentary rocks, which include alluvial deposits as the main aquifer unit, with variable permeability, high transmisivity and high hydraulic conductivity. Through the chemical composition of water, two different groundwater units were detected and are classified as calciumbicarbonate and chloridesodiummagnesiumbicarbonate. Helping by geophysical exploration using electrical resistivity, information is obtained from the aquifer, as the depth of water level, lateral and in depth variations of the alluvial deposits.

Key words: Quepos, Naranjo river, Naranjito aquifer, hydrogeology, hidrogeochemestry.
\end{abstract}




\section{INTRODUCCIÓN}

La zona de estudio se localiza en la cuenca baja del río Naranjo, en el sector de Quepos en la provincia de Puntarenas y cubre un área de 41,6 $\mathrm{km}^{2}$ (Fig. 1). Por tratarse de una región costera, los acuíferos de mayor producción quedan restringidos principalmente a las formaciones aluviales.

Debido al crecimiento demográfico que ha sufrido el cantón de Aguirre, posterior a la construcción de la carretera costanera en la zona sur, ha aumentado considerablemente la demanda del recurso hídrico, lo que ha obligado la búsqueda de nuevas fuentes de suministro. El acuífero aluvial Naranjito representa actualmente una de las tres fuentes de abastecimiento de agua potable de la comunidad de Quepos y otras comunidades cercanas, y es por esta razón que se ha planteado como objetivo principal, caracterizar los parámetros hidrogeológicos e hidrogeoquímicos.

La metodología de trabajo consistió en la recopilación y análisis de información bibliográfica, así como la aplicación de técnicas de campo como la exploración geofísica, con el método de resistividad eléctrica tipo sondeo eléctrico vertical, prueba de bombeo, muestreo y análisis de aguas, con la finalidad de elaborar un modelo hidrogeológico conceptual del acuífero.

Existen varios estudios geológicos previos que muestran diversos rasgos geomorfológicos, estratigráficos y estructurales, la mayoría de los cuales se realizaron sobre el Promontorio de Quepos. Se destaca los trabajos de Malavassi (1961), Henningsen (1965), Acuña (1975), Bergoeing \& Jiménez (1978), Azema et al. (1979), Schmidt-Effing (1979), Granados \& Aguilar (1983), Baumgartner et al. (1984), Drake (1989) y Arias (2000). Son pocos los trabajos hidrogeológicos que se han desarrollado en la zona, reconociendo el estudio realizado por SENARA (2003), donde se describen algunos parámetros hidráulicos del acuífero Naranjito, junto con una evaluación preliminar de la vulnerabilidad a partir de la metodología GOD.

Esta investigación es un extracto del proyecto de graduación en el cual se valoran características

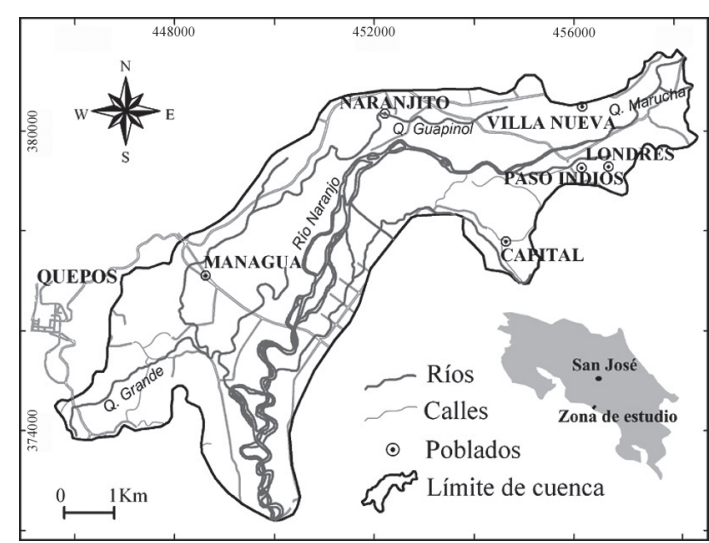

Fig. 1: Ubicación de la zona de estudio

geofísicas, potencial hídrico y vulnerabilidad intrínseca del acuífero a partir de la metodología DRASTIC.

\section{ASPECTOS GEOLÓGICOS}

La geología está compuesta por rocas ígneas y sedimentarias del Terciario Inferior y por depósitos aluviales Cuaternarios. Las distintas unidades aflorantes fueron correlacionadas con formaciones formales, agrupando las similitudes de las litologías, principales características estratigráficas y sedimentológicas, ambientes de depositación y génesis, contenido fosilífero, entre otras particularidades, las cuales se describen de la más antigua a la más reciente (Fig. 2).

El basamento regional lo constituye el Sub Complejo Basáltico del Promontorio de Quepos, al cual lo sobreyace la Formación (Fm.) Caótica Punta Quepos, posteriormente son depositados los conglomerados y areniscas calcáreas de la Fm. Calcarenita Punta Serrucho y las lutitas y areniscas calcáreas de la Fm. Térraba, completando la secuencia estratigráfica con los depósitos Cuaternarios, que han sido subdivididos como antiguos y recientes, reconociéndose en este último tres terrazas no cíclicas denominadas Londres, Paso Indios y La Managua. Estructuralmente, el área está dominada por fallas inversas como la Falla Longitudinal de Costa Rica, a la cual se asocian fallas normales con componente de desplazamiento de rumbo. 


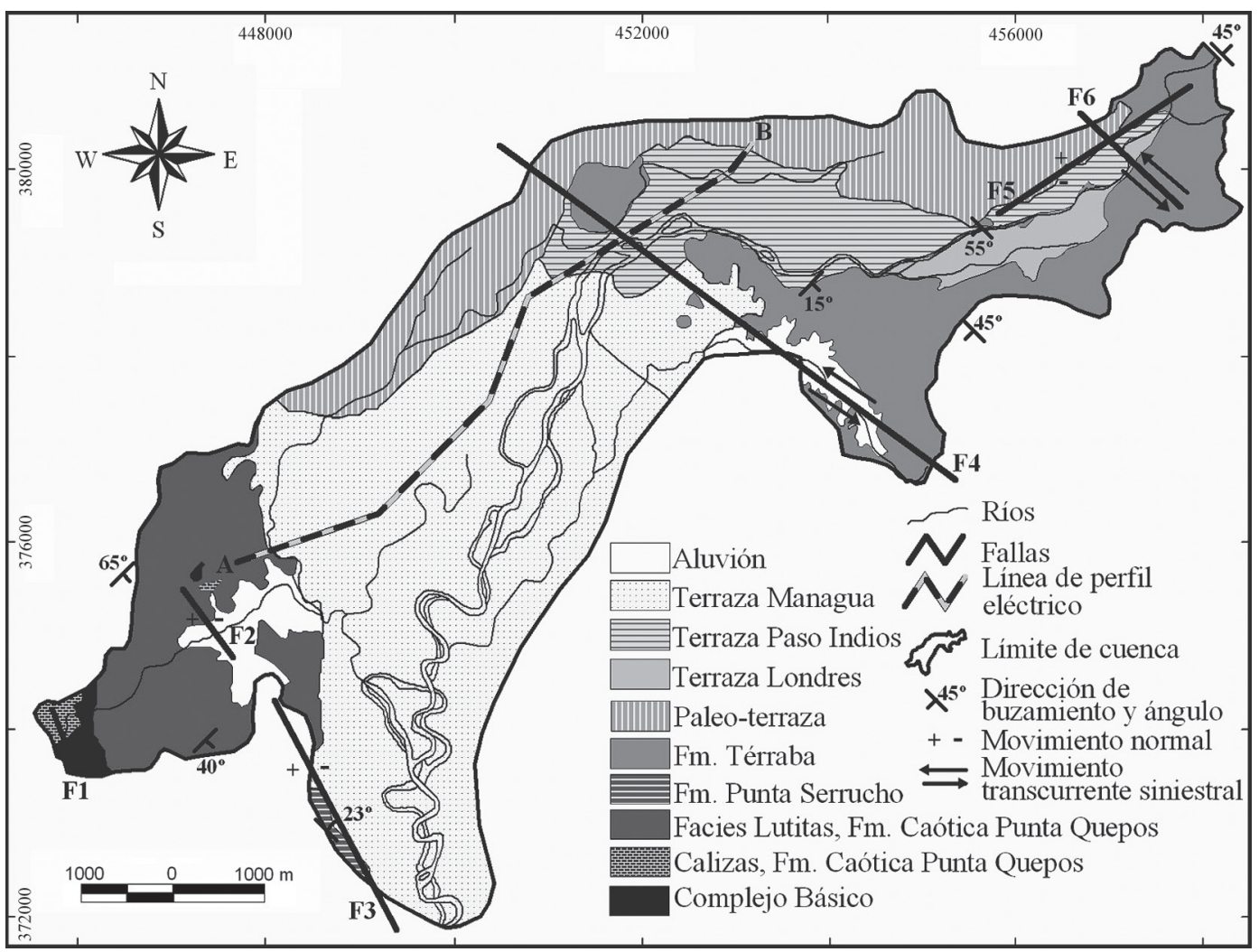

Fig. 2: Mapa geológico de la cuenca baja del río Naranjo. Se muestra las 3 terrazas de la Unidad de Depósitos Aluviales Recientes. Falla observada (F2). Fallas inferidas o fotointerpretadas (F1, F3, F4, F5 y F6). Falla Longitudinal de movimiento inverso con componente de desplazamiento de rumbo siniestral (F4). La línea de perfil AB corresponde al perfil de correlación geoeléctrico

\section{Sub - Complejo Basáltico del Promontorio de Quepos}

El promontorio de Quepos está constituido por un grupo de cerros aislados, en los cuales aflora un complejo básico, cuya edad se encuentra entre el Paleoceno Tardío al Eoceno Temprano. Este límite fue interpretado por las mezclas de rocas que se observan al oeste de la Playa Espadilla, como resultado de eventos volcánicos (Schmitd-Effing, 1979); por otro lado Azema et al. (1979) reconocieron microfósiles del Daniano en calizas pelágicas asociadas a lavas que se encuentran en Playa Espadilla - Punta Quepos.

Estratigráficamente, se encuentra sobreyacido por brechas y conglomerados de la Fm. Caótica Punta Quepos y por una secuencia sedimentaria del Terciario.

\section{Formación Caótica Punta Quepos}

Está compuesta por clastos del complejo básico, brechas y conglomerados de la Formación Brecha Puerto Carrillo, lutitas y areniscas silíceas (turbiditas distales de la Formación Lutita - Areniscas Cabo Blanco), areniscas y lutitas calcáreas y calcarenitas someras, las cuales se encuentran mezcladas y deformadas sinsedimentariamente con las "calizas de Quepos". Las calizas predominan en esta formación y están dispuestas como bloques métricos hasta cuerpos lentiformes, en una matriz de lutitas silíceas y calcáreas con abundante microfauna de radiolarios y foraminíferos planctónicos (Baumgartner et al., 1984).

Debido a las deformaciones tectónicas y sedimentarias ocurridas en este sector del promontorio, 
estos depósitos turbidíticos se presentan con abundantes fracturas y capas irregulares, por lo que no se visualiza ningún tipo de estratificación ni estructuras sedimentarias. La dirección de buzamiento local preferencial es hacia el SE, con inclinaciones que oscilan entre los $30^{\circ}$ y $40^{\circ}$. A lo largo de la Quebrada Grande se reconocen varios saltos de falla donde se evidencia un movimiento de tipo normal con rumbo NW-SE (F2 de la Fig. 2).

Se reconoce un espesor máximo calculado en la zona de estudio de $1400 \mathrm{~m}$, el cual se obtuvo tomando el criterio de inclinación preferencial de las capas.

Baumgartner et al. (1984) caracterizan el contacto basal por presentar un cizalle importante, con el cual es posible concluir que esta formación es producto de un emplazamiento por gravedad de sedimentos poco litificados, a partir de la combinación de deslizamientos y flujos de detritos. La edad de los materiales según estos mismos autores es del Eoceno Temprano / Medio.

\section{Formación Calcarenita Punta Serrucho}

Conformada por intercalaciones de areniscas finas y medias, dispuestas en laminación paralela de aproximadamente $1 \mathrm{~cm}$ de espesor. Presenta cemento calcáreo con variaciones de color de crema a gris, con estructuras sedimentarias de tipo gradación positiva, laminación paralela, estratificación cruzada, slumps, entre otras (Valerín, 1982). El espesor máximo observado es de $40 \mathrm{~m}$.

Geomorfológicamente, estos materiales se encuentran dispuestos en cerros alargados en dirección NW-SE, debido principalmente a que corresponden a la dirección estratigráfica de las rocas sedimentarias, y además al fuerte control estructural provocado por la falla Quepos (F3 de la Fig. 2), la cual es una falla normal con una componente de tipo transcurrente dextral (Denyer et al., 2003). La dirección de inclinación predominante de las rocas es hacia el NE, con un ángulo promedio de $23^{\circ}$.

Se define una edad del Eoceno Medio, a partir de la asociación de macroforaminíferos (Malavassi, 1961 y Azema et al., 1979) y del Eoceno Superior a partir de muestras del conglomerado basal y de la parte intermedia (Baumgartner et al., 1984).

\section{Formación Térraba}

Está constituida por lutitas grises oscuras hasta café claro cuando se encuentra muy meteoriza$\mathrm{da}$, que generalmente presentan cemento carbonatado. Se reconocen 3 facies: la primera se compone de areniscas calcáreas de grano fino a medio de color beige, dispuestas en estratos centimétricos, que corresponde con depósitos turbidíticos intermedios, los cuales se caracterizan por presentar gradación positiva. La segunda facies corresponde con lutitas arenosas de color gris claro, la cual presenta una ligera laminación paralela, así como un bajo contenido de macrofósiles de tipo gastrópodos. La tercera facies se compone de areniscas con un alto porcentaje de moldes de macrofósiles de tipo principalmente gastrópodos de $1,5 \mathrm{~cm}$ de longitud como máximo, además se observan en menor cantidad bivalvos de $1 \mathrm{~cm}$ de longitud, se encuentra muy alterada por lo que presenta una coloración amarillenta, así como pátinas de óxidos de hierro, y debido al grado de meteorización el cemento carbonatado es menos evidente.

Esta unidad presenta planos de estratificación laminar planar bien definidos, de espesores centimétricos a métricos, con incrustaciones de calcita entre los estratos, así como pátinas de manganeso.

Estructuralmente, los cerros donde aflora esta unidad se encuentran disectados por líneas de falla, tal como ocurre con la Falla Longitudinal (F4 de la Fig. 2), la cual presenta un movimiento inverso con componente transcurrente siniestral (Acuña, 1975; Bergoeing \& Jiménez, 1978; Drake, 1989 y Denyer et al., 2003). Además, a nivel local se observa un fuerte fracturamiento de la roca, lo cual queda evidenciado por las múltiples fracturas conjugadas, generando un aspecto friable.

Existen variaciones en la dirección de inclinación, producto de la génesis de estas rocas por motivo de los deslizamiento sinsedimentarios, de manera que se reconocen direcciones de buzamientos hacia el SE, SW y NE con variación de ángulos que van desde los $15^{\circ}$ hasta $\operatorname{los} 75^{\circ}$ (Fig. 2). 
Esta formación tiene una edad de Oligoceno - Mioceno Inferior, según Mora (1979).

\section{Unidad Depósitos Aluviales Antiguos}

Esta unidad se reconoce como un abanico aluvial con una secuencia de gradación positiva, cuya capa inferior está compuesta por depósitos con clastos de grava gruesa redondeada de aproximadamente $10 \mathrm{~cm}$, englobados en una matriz de arenas gruesas y arcilla. La capa superior se caracteriza por estar compuesta de grava media, un mayor porcentaje de matriz fina y clastos ligeramente imbricados hacia el norte.

Se caracteriza y a la vez se diferencia de los depósitos aluviales recientes por la coloración rojiza y textura arcillosa producto de la meteorización, así como por el mayor porcentaje de matriz arcillosa de alta plasticidad, la cual engloba los clastos redondeados y subredondeados centimétricos a decimétricos.

Debido a los diferentes eventos de levantamiento tectónicos ocurridos en la zona, se reconoce la formación de una paleo-terraza levantada hasta aproximadamente $40 \mathrm{~m}$ del nivel del suelo, la cual se observa al NE del poblado Paso Indios. Este escarpe representa una posible traza de falla de tipo normal, la cual provoca los movimientos verticales (F5 de la Fig.2).

En esta terraza, denominada por Drake (1989) como Aluvión Naranjito, se encuentran los poblados de Villa Nueva y Naranjito, que de acuerdo con la fotointerpretación y lo observado en el campo, disminuye su espesor desde el sector NE, hacia el río Paquita, pasando de un espesor de $40 \mathrm{~m}$ a $5 \mathrm{~m}$.

\section{Unidad Depósitos Aluviales Recientes}

Afloran en las partes topográficamente más bajas, a lo largo del río Naranjo. Está constituida por depósitos aluviales, los cuales fueron transportados y depositados principalmente por el río Naranjo en las llanuras de inundación. De forma general, se encuentran materiales de todo tipo de granulometrías, dispuestos en capas o como relleno de paleocanales.
El sistema de drenaje se caracteriza por ser meándrico; sin embargo, conforme se acerca al litoral, se produce una combinación de patrones meándrico y entrelazado. Es típico encontrar en esta zona morfologías de meandros abandonados, lagunas en media luna, islas de depositación, barras arenosas o point bars, malecones y pantanos cerca de los malecones.

En esta unidad al igual que la anterior, se observan terrazas no cíclicas o disparejas a diferentes niveles de elevación, lo cual evidencia movimientos neotectónicos. Se reconocieron al menos tres terrazas principales:

\section{Terraza Londres}

Se localiza en el poblado de Londres y presenta una elevación a nivel del cauce del río de aproximadamente $20 \mathrm{~m}$. Está constituida por depósitos aluviales gruesos, los cuales se caracterizan por presentar bloques métricos en la superficie de la terraza, y se encuentran dispersos de forma irregular por todo el poblado de Londres. En la parte inferior de la terraza se observan capas de grava de $20 \mathrm{~cm}$ a $30 \mathrm{~cm}$, con clastos subredondeados y con buena grano selección, los cuales se encuentran imbricados. La parte superior se reconoce por presentar depósitos de gravas de $2 \mathrm{~cm}$ a $15 \mathrm{~cm}$ de diámetro con moderada a baja grano selección.

\section{Terraza Paso Indios}

Se ubica en las planicies del poblado de Paso Indios, al norte del área de estudio. Consiste en una planicie elongada en dirección E-W, en donde se reconocieron tres niveles de terrazas distintas, dos de las cuales son discontinuas y no superan los $3 \mathrm{~m}$ de altura. La tercera terraza se observa en el borde actual del cauce del río Naranjo, que debido a la constante erosión es posible detallar las diferentes capas que la componen. Las cuales varían lateralmente conforme nos acercamos al ápice de la cuenca baja, pasando de depósitos de grava media a gruesa de mala selección, con matriz de grava fina y arena gruesa cementada a depósitos de grava bien seleccionados y con 
contactos bien diferenciados. Esta unidad se caracteriza por ser de una permeabilidad aparente buena, ya que se logra observar diversos brotes de agua en su base.

De los afloramientos observados en esta terraza se concluye que existe un incremento en el espesor visible conforme se asciende topográficamente, además, que el porcentaje de matriz observado disminuye, debido principalmente a las fuertes corrientes de depositación que logran transportar todos los materiales finos, y que posteriormente son depositados en las zonas bajas, como se observa en los depósitos de la Terraza La Managua.

\section{Terraza La Managua}

Se ha denominado así a toda la extensión restante de la llanura aluvial, y cuyos afloramientos cerca del cauce del río Naranjo son escasos debido a la fuerte erosión y poca sedimentación de materiales gruesos. Se reconocen depósitos finos mezclados con cantos redondeados a subredondeados de mala selección, los cuales se encuentran flotantes en una matriz arcillosa de color café rojiza, y que por lo general presentan imbricación hacia el NE.

Estos depósitos son más finos que los encontrados en las terrazas de Londres y Paso Indios. Los materiales de grava más gruesos oscilan entre los $3 \mathrm{~cm}$ hasta $15 \mathrm{~cm}$ de diámetro, los cuales han sido depositados en capas decimétricas producto de eventos de mayor carga y turbulencia del río Naranjo. Se reconocen capas limo arcillosas hasta arenosas intercaladas con algunas capas más gruesas que por lo general tienden a acuñarse.

Dentro de esta terraza se localiza los poblados de Finca Managua y Llamarón, así como el campo de aterrizaje y el Hospital de Quepos.

\section{CARÁCTERÍSTICAS GEOFÍSICAS E HIDROGEOLÓGICAS}

La cuenca baja del río Naranjo, corresponde a una zona donde abundan los depósitos aluviales compuestos por gravas de diferentes granulometrías, así como lentes de arenas y arcillas, los cuales originan geometrías irregulares, tanto en capas, como rellenos de canales por donde el agua subterránea se moviliza.

Debido a estas irregularidades de la zona, se elaboró una exploración geofísica mediante la metodología de resistividad eléctrica tipo sondeo eléctrico vertical (SEV). A partir de 14 sondeos realizados se construyeron 6 perfiles geoeléctricos con el fin de determinar el modelo conceptual, tanto la profundidad y espesores, así como los límites del acuífero, a partir de la correlación de las distintas capas geoeléctricas, utilizando como guía la descripción litológica de algunos pozos. Se utilizó el dispositivo eléctrico Schlumberger, con una abertura máxima de los electrodos $\mathrm{AB} / 2$ de $500 \mathrm{~m}$, con una profundidad máxima de investigación teórica de 125m (Telford et. al., 1996). Para esta publicación se muestra solamente el perfil A - B el cual atraviesa de forma transversal a la zona investigada (Fig 2 y 3 ).

En este perfil mostrado en la figura 3, se ha determinado una secuencia de 6 capas geoeléctricas. El basamento está constituido por una capa de resistividad de 180 a $195 \Omega \mathrm{m}$, el cual fue observado en los SEV 1, SEV 5, SEV 9 y SEV 14. Esta capa se correlaciona con depósitos de arenas finas. Esta misma capa geoeléctrica se reconoce además, como un lente y como depósito de relleno superficial, los cuales presentan valores de resistividad de 120 a $150 \Omega \mathrm{m}$ y con espesores de 1,3 a $3 \mathrm{~m}$ respectivamente.

Sobre el basamento se tienen 5 capas geoeléctricas distintas, cuyos valores de resistividad varían entre 230 a mayor de $3765 \Omega \mathrm{m}$. Esta variación lateral se debe principalmente a la génesis de depositación de los materiales aluviales, donde se muestra en la figura 3 la gradación granulométrica, teniendo los materiales más finos en la tarraza La Managua y los más gruesos en la parte central y superior de la cuenca hacia la Terraza Paso Indios.

Debido a que se trata de un perfil longitudinal a la cuenca, se destacan una serie de transiciones en los depósitos gravosos, donde existen depósitos de grava con arena gruesa hacia el SW del perfil en el sector topográficamente más bajo, cuya resistividad es de $380 \Omega \mathrm{m}$ y un espesor de $9 \mathrm{~m}$. 


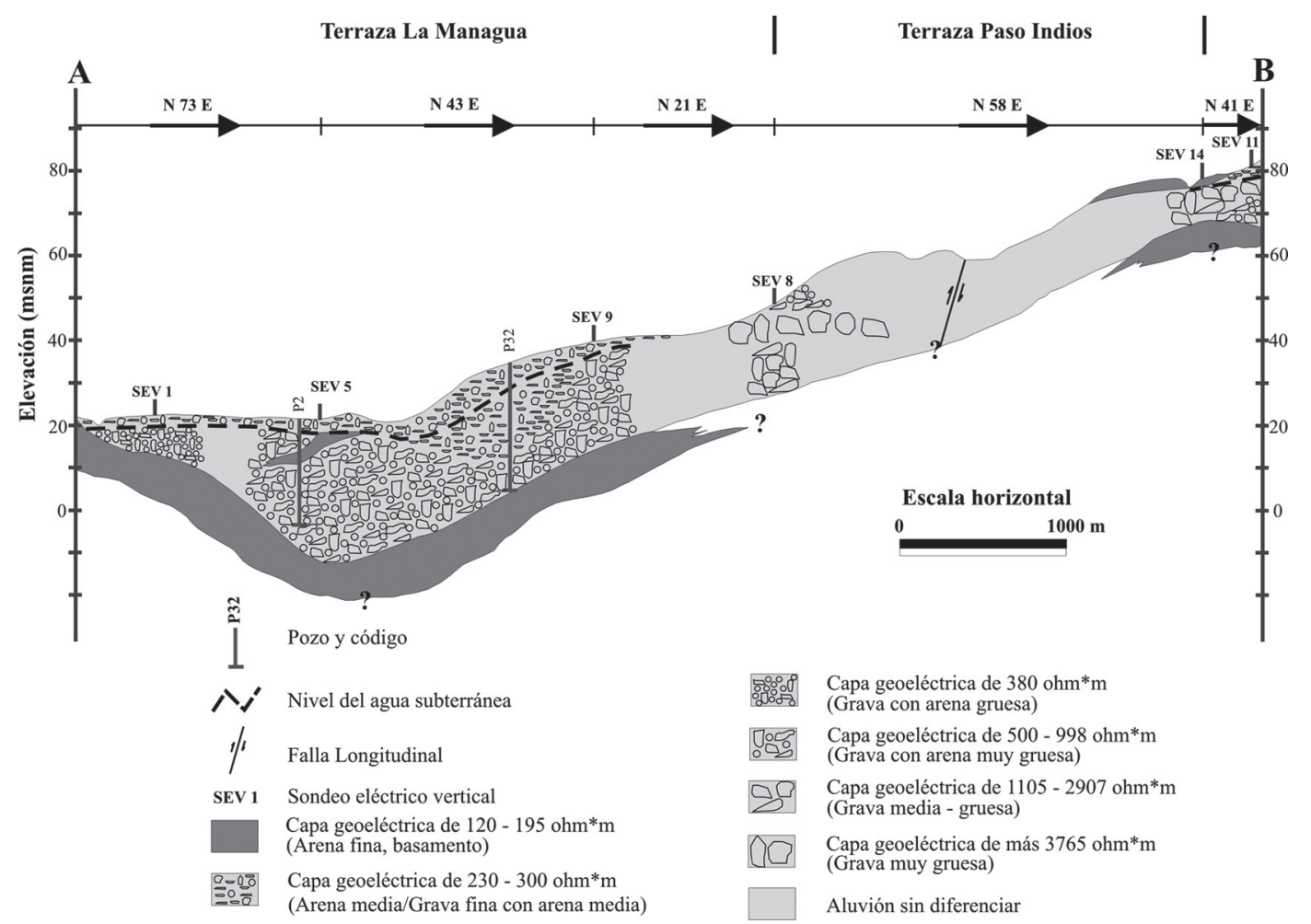

Fig. 3: Perfil de correlación geoeléctrico $\mathrm{AB}$ en donde se muestra la relación entre la conductividad eléctrica y las distintas granulometrías

En la parte central del perfil, se asocia un depósito de grava con arena muy gruesa, cuyo valor de resistividad oscila entre 500 y $585 \Omega \mathrm{m}$ y presenta un espesor máximo $28 \mathrm{~m}$ el cual se observa en el SEV 5 y SEV 9. Otras capas de igual litología se correlacionan a nivel superficial en el SEV8 con una resistividad de $998 \Omega \mathrm{m}$, y la última capa del SEV 11 con una resistividad de $607 \Omega \mathrm{m}$.

A partir del SEV 8 y hacia el NE del perfil, se observan depósitos de grava media a gruesa, cuya resistividad varía entre 1105 a $2907 \Omega \mathrm{m}$ con un espesor mínimo de 10,5 m. En este mismo sondeo se identifica un lente de resistividad de más de $3765 \Omega \mathrm{m}$, el cual se correlaciona con gravas muy gruesas y un espesor de 7,8 m. Ambos materiales se caracterizan por ser depósitos limpios, es decir, donde el porcentaje de matriz es muy bajo a nulo. Cabe destacar que la capa de mayor resistividad se asocia a la falta de agua subterránea, ya que estos depósitos reciben una percolación directa hacia las capas subyacentes.
Finalmente, se identificó una capa de resistividad de 230 a $300 \Omega \mathrm{m}$, la cual corresponde con depósitos de gravas finas con arenas medias. $\mathrm{Su}$ espesor varía considerablemente de $3 \mathrm{~m}$ hasta $23 \mathrm{~m}$ a nivel del pozo P32 y de acuerdo con los sondeos realizados. Esta capa forma parte de la llanura de inundación actual de la Terraza La Managua.

En este perfil se identifican dos niveles de saturación: al SW del perfil se encuentra a una profundidad aproximada de $2 \mathrm{~m}$ y hacia en el sector central, el nivel se localiza aproximadamente entre los 5 y $6,5 \mathrm{~m}$. A la altura del SEV 11 se reconoce un nivel a una profundidad aproximada de $5 \mathrm{~m}$, que corresponde con la Unidad de Depósitos Aluviales Antiguos.

A partir de la profundidad del nivel freático y el espesor de los aluviones, se determinó el espesor saturado del acuífero, el cual se muestra en la figura 4. Este espesor oscila entre 1 y $36 \mathrm{~m}$, siendo en la zona central del área donde se encuentra 


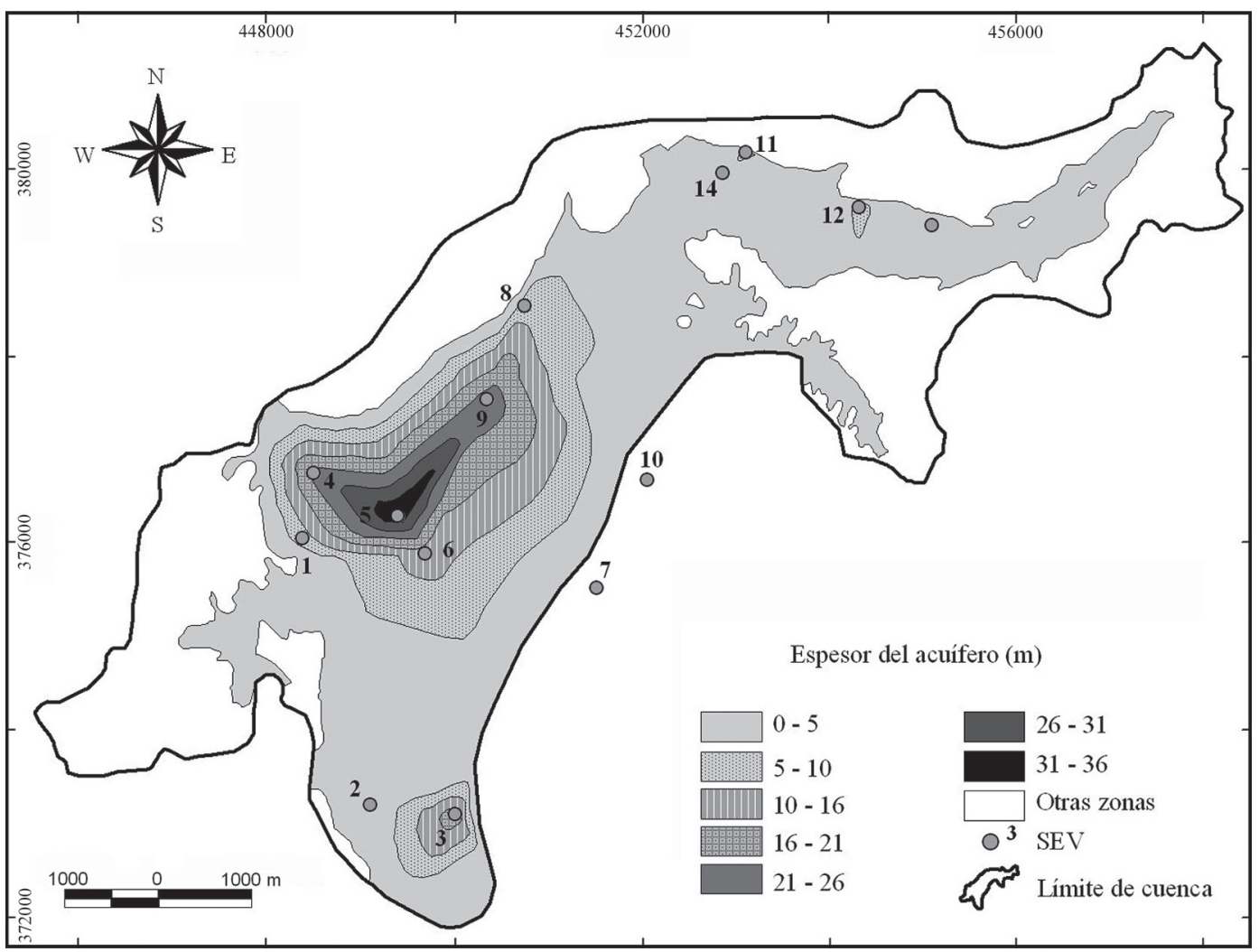

Fig. 4: Distribución de espesores del Acuífero Aluvial Naranjito y ubicación de los sondeos eléctricos verticales

el mayor espesor, precisamente en los alrededores de los pozos del AyA. Esta zona es la más adecuada para la perforación de futuros pozos para el abastecimiento público, manteniendo una distancia adecuada para evitar interferencia.

En la zona de investigación existen 42 pozos de los cuales 25 son perforados y 17 son excavados. De éstos, 8 pozos captan los Depósitos Aluviales Antiguos, 1 en el Complejo Básico, 5 en la Formación Caótica Punta Quepos y 28 en los Depósitos Aluviales Recientes (Fig. 5, Cuadro 1). Los principales pozos perforados son utilizados para abastecimiento público y turístico, por lo que los tiempos de bombeo son de mayor continuidad que los pozos excavados.

Se han identificado dos unidades hidrogeológicas, la Unidad de Depósitos Cuaternarios Antiguos y la Unidad de Depósitos Cuaternarios
Recientes, denominada esta última como Acuífero Aluvial Naranjito, con base a los criterios establecidos por la ASTM (2004) y representa la principal unidad hidrogeológica del área.

Con el propósito de determinar los parámetros hidrogeológicos del Acuífero Aluvial Naranjito, se realizó una prueba de bombeo de 19 horas a caudal contante de $5 \mathrm{l} / \mathrm{s}$, en el pozo artesanal perteneciente a la empresa Excavaciones Victoria (P11), la cual se localiza en los Depósitos Aluviales Recientes y cercano a los pozos de AyA. El nivel estático inicial fue de 2,07 m.b.n.s, y el dinámico final de 2,51 m.b.n.s, para un abatimiento de $0,44 \mathrm{~m}$. Según la gráfica de bombeo mostrada en la figura 6 , se reconoce una sección de aporte de agua o barrera positiva entre los ciclos de 10 y 100 representado por la línea constante donde no hubo abatimiento, posterior a los 180 min se genera una barrera negativa 


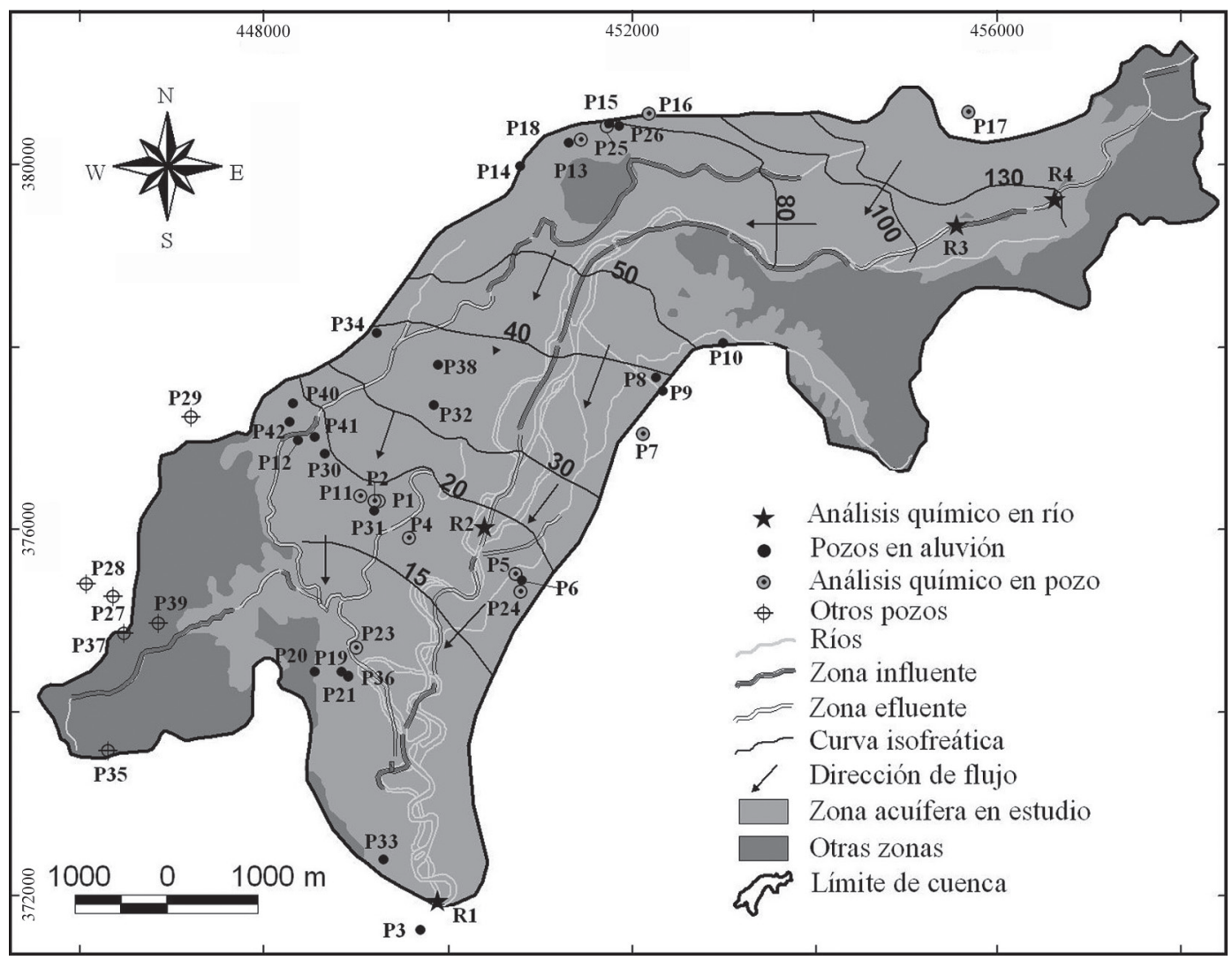

Fig. 5: Mapa hidrogeológico. Se muestra las líneas equipotenciales y dirección de flujo del agua subterránea, ubicación de los pozos y ubicación de los sitios de muestreo hidrogeoquímico y las zonas de influencia y efluencia del río Naranjo.

muy leve, producto de las irregularidades geométricas y granulométricas de los depósitos sedimentarios, donde varían las condiciones hidráulicas.

Mediante las ecuaciones de Papadopulus Cooper (Kruseman \& De Ridder, 1994), cuyo método es utilizado para calcular los parámetros hidráulicos en pozos de gran diámetro, se determinó un valor de transmisividad de $781 \mathrm{~m}^{2} / \mathrm{d}$, con un coeficiente de almacenamiento de 0,1 indicativo de acuíferos libres, aunque en algunos sectores tienda ser semi confinado. Considerando un espesor del acuífero promedio según la figura 4 de $18 \mathrm{~m}$ se obtiene una conductividad hidráulica de $43 \mathrm{~m} / \mathrm{d}$, equivalente a $4,97 \times 10^{-2} \mathrm{~cm} / \mathrm{s}$; aunque considerando el espesor máximo encontrado de $36 \mathrm{~m}$, localizado en la parte central de la cuenca donde se localizan los pozos de AyA, el valor de conductividad es de
$22 \mathrm{~m} / \mathrm{d}$, equivalente a $2,54 \times 10^{-2} \mathrm{~cm} / \mathrm{s}$, ambos correspondiendo según la clasificación de Freeze \& Cherry (1979) como acuíferos gravosos.

Dado que ambas unidades hidrogeológicas son de tipo libre, se ha utilizado para la elaboración del mapa de curvas isofreáticas de la zona, la información de los niveles estáticos obtenidos en el campo. En los sectores donde no hay información, principalmente en la parte baja, se utilizó el criterio de flujo base de los ríos, determinado a partir de diferentes puntos de elevación a lo largo del cauce. Como se observa en la figura 5, en la parte superior de la cuenca, el agua subterránea se mueve en una dirección aproximada SW a $\mathrm{E}-\mathrm{W}$ con un gradiente hidráulico promedio de 0,04 ; mientras en la parte media y baja el agua se mueve hacia el S -SW en dirección hacia el mar, 
Cuadro 1

Características de pozos en el área de estudio

\begin{tabular}{|c|c|c|c|c|c|c|c|}
\hline Pozo & $\mathrm{N}$ & E & Prof. (m) & N.E. (m) & $\begin{array}{c}\text { Elev. NE } \\
\text { (msnm) }\end{array}$ & $\mathrm{Q}(\mathrm{l} / \mathrm{s})$ & $\begin{array}{l}\text { Unidad } \\
\text { captada }\end{array}$ \\
\hline P1 & 376300 & 449250 & 25,00 & 3,44 & 19,6 & 25 & DR \\
\hline P2 & 376300 & 449200 & 25,00 & & 19,3 & 25 & DR \\
\hline $\mathrm{P} 3$ & 371623 & 449702 & & 4,67 & 15,0 & & DR \\
\hline $\mathrm{P} 4$ & 375902 & 449581 & & 4,86 & 18,5 & & DR \\
\hline P5 & 375504 & 450743 & 3,62 & 2,42 & 18,7 & & DR \\
\hline P6 & 375442 & 450813 & & & 18,5 & & DR \\
\hline $\mathrm{P} 7$ & 377038 & 452136 & 9,00 & 3,58 & 43,0 & & DR \\
\hline $\mathrm{P} 8$ & 377655 & 452272 & & 7,72 & 45,0 & & DR \\
\hline P9 & 377510 & 452347 & & 7,10 & 46,0 & & DR \\
\hline P10 & 378027 & 453009 & & 5,11 & 59,0 & & DR \\
\hline P11 & 376359 & 449049 & 4,34 & 3,71 & 20,0 & & DR \\
\hline $\mathrm{P} 12$ & 376966 & 448368 & & & 20,0 & & DR \\
\hline P13 & 380249 & 451459 & & 5,51 & 80,0 & & DA \\
\hline P14 & 379957 & 450791 & & & 73,8 & & DA \\
\hline P15 & 380429 & 451764 & 4,00 & 0,00 & 84,5 & & DA \\
\hline P16 & 380529 & 452194 & 9,00 & 9,61 & 94,0 & & DA \\
\hline P17 & 380550 & 455683 & 10,00 & 4,04 & 174,4 & & DA \\
\hline P18 & 380215 & 451321 & 11,00 & 9,00 & 76,0 & & DA \\
\hline P19 & 374443 & 448843 & 2,95 & 1,81 & 16,8 & & DR \\
\hline P20 & 374444 & 448552 & 3,75 & 1,19 & 34,6 & & DR \\
\hline $\mathrm{P} 21$ & 374394 & 448922 & 2,70 & 1,91 & 13,5 & & DR \\
\hline $\mathrm{P} 22$ & 374395 & 448919 & & & 13,7 & & DR \\
\hline $\mathrm{P} 23$ & 374704 & 449006 & & & 10,8 & & DR \\
\hline $\mathrm{P} 24$ & 375316 & 450794 & & & 13,7 & & DR \\
\hline $\mathrm{P} 25$ & 380392 & 451740 & 85,00 & 4,85 & 81,0 & & DA \\
\hline P26 & 380404 & 451876 & 80,00 & 3,35 & 83,0 & & DA \\
\hline P27 & 375268 & 446344 & 40,00 & 4,28 & 47,0 & 1 & $\mathrm{CB}$ \\
\hline P28 & 375406 & 446056 & 44,00 & 14,60 & & 0,45 & $\mathrm{CB}$ \\
\hline P29 & 377225 & 447200 & 35,00 & 13,68 & 128,0 & 1 & $\mathrm{~L}$ \\
\hline P30 & 376820 & 448660 & & & 20,0 & & DR \\
\hline P31 & 376200 & 449200 & 30,00 & & 13,0 & 22 & DR \\
\hline P32 & 377350 & 449850 & 30,00 & & 22,7 & & DR \\
\hline P33 & 372400 & 449300 & 20,00 & & & & DR \\
\hline P34 & 378140 & 449225 & & & & & DA \\
\hline P35 & 373585 & 446295 & 60,00 & & 149,3 & & $\mathrm{CB}$ \\
\hline P36 & 374400 & 448920 & 25,00 & & 13,8 & 1 & DR \\
\hline P37 & 374866 & 446466 & 120,00 & & 120,0 & 2 & $\mathrm{~L}$ \\
\hline
\end{tabular}


Cuadro 1 (continuación)

Características de pozos en el área de estudio

\begin{tabular}{cccccccc}
\hline Pozo & N & E & Prof. $(\mathrm{m})$ & N.E. (m) & $\begin{array}{c}\text { Elev. NE } \\
(\mathrm{msnm})\end{array}$ & Q (1/s) & $\begin{array}{c}\text { Unidad } \\
\text { captada }\end{array}$ \\
\hline P38 & 377800 & 449900 & 25,00 & & 37,8 & 1 & DR \\
P39 & 374978 & 446839 & 73,00 & 50,00 & 62,7 & 1 & L \\
P40 & 377350 & 448250 & 30,00 & 8,00 & 20,0 & 1 & DA \\
P41 & 377000 & 448500 & 34,00 & 2,00 & 20,0 & 1,25 & DR \\
P42 & 377150 & 448270 & 30,00 & 3,00 & 20,0 & & DR,L \\
\hline
\end{tabular}

Fuente: Archivo de pozos, SENARA, 2007 y levantamiento de campo

Lat.: latitud; Long.: longitud; Prof.: profundidad; NE: nivel estático; Elev. NE: elevación del nivel estático; DA: Depósitos Antiguos; DR: Depósitos Recientes; L: Lutitas; CB: Complejo Básico

con un gradiente hidráulico promedio de 0,007. Es importante destacar que el flujo subterráneo sigue una trayectoria paralela al río Naranjo, y en algunos sectores se indica una dirección de descarga hacia el río y quebradas de la cuenca, mostrando de esta manera una relación hidráulica de influencia y efluencia entre el agua superficial y subterránea.

El límite entre ambos acuíferos se reconoce a partir de una diferencia topográfica, química y litológica. Se asume una conexión hidráulica entre el acuífero de los depósitos aluviales antiguos hacia el Acuífero Naranjito, ya que no se observó ninguna descarga de agua. Por otro lado el límite del Acuífero Naranjito queda definido por el contacto entre las formaciones. En el caso del límite Este, no existen evidencias geomorfológicas ni topográficas claras que separen al acuífero adyacente, por ser parte de una llanura aluvial muy plana.

Se realizaron 30 pruebas de infiltración utilizando la metodología de los anillos concéntricos (Custodio \& Llamas, 1983) con una duración de dos horas cada una. Las mismas se llevaron a cabo en distintos puntos del área estudiada, con el propósito de determinar la velocidad de infiltración en las diferentes unidades hidrogeológicas. Además, se tomó muestra de suelo en el sitio de la prueba para relacionar el tipo de suelo con el valor obtenido de infiltración.

Las capas de suelo que cubre este acuífero presentan una velocidad de infiltración promedio de $0,42 \mathrm{~m} / \mathrm{d}$, las cuales varían entre $1,88 \mathrm{~m} / \mathrm{d}$ y $0,01 \mathrm{~m} / \mathrm{d}$. Estos valores son variables debido a las características de la superficie donde se realizó la prueba, ya que existen sectores donde predominan materiales limo-arcillosos producto de los desbordamientos del río durante eventos de crecidas, por lo que la infiltración tiende a disminuir. Esta característica del terreno evidencia la poca infiltración directa que se genera en estas planicies aluviales, por lo que se determinó que la principal recarga de este acuífero es a través de los depósitos gruesos del río Naranjo, evidenciado mediante los caudales diferenciales obtenidos a lo largo de este río (Fig. 5). En esta figura se muestra las principales áreas donde existe una recarga del río hacia las zonas influentes.

\section{CARACTERÍSTICAS HIDROGEOQUIIMICAS}

Se realizaron 16 muestreos de agua para análisis físico - químicos, los cuales fueron recolectados en los pozos del AyA, pozos excavados y en diferentes secciones del río Naranjo y fueron analizados por el Laboratorio Nacional de Aguas del AyA (Cuadro 2). El uso actual del agua subterránea principalmente es para consumo humano, mientras que el agua superficial es tomada en algunas zonas para el riego de plantaciones especialmente de palma africana.

A partir de los resultados obtenidos se elaboró un diagrama de Piper para todas las muestras, y diagramas de Stiff para cada muestra en particular, para clasificar el tipo hidrogeoquímico del agua e identificar las relaciones existentes entre el agua subterránea y superficial. 
Cuadro 2

Composición química del agua recolectada en la zona de estudio

\begin{tabular}{cccccccccccc}
\hline Pozo & $\mathrm{pH}$ & $\begin{array}{c}\text { Conductividad } \\
\text { eléctrica } \\
(\mathrm{mS} / \mathrm{cm})\end{array}$ & $\begin{array}{c}\text { Alcalinidad } \\
\text { total } \\
(\mathrm{mg} / \mathrm{l})\end{array}$ & $\begin{array}{c}\text { Dureza } \\
\text { total } \\
(\mathrm{mg} / \mathrm{l})\end{array}$ & $\begin{array}{c}\mathrm{Ca}^{2+} \\
(\mathrm{mg} / \mathrm{l})\end{array}$ & $\begin{array}{c}\mathrm{Mg}^{2+} \\
(\mathrm{mg} / \mathrm{l})\end{array}$ & $\begin{array}{c}\mathrm{Na}^{+} \\
(\mathrm{mg} / \mathrm{l})\end{array}$ & $\begin{array}{c}\mathrm{HCO}^{3-} \\
(\mathrm{mg} / \mathrm{l})\end{array}$ & $\begin{array}{c}\mathrm{SO}_{4}^{2-} \\
(\mathrm{mg} / \mathrm{l})\end{array}$ & $\begin{array}{c}\mathrm{NO}^{3-} \\
(\mathrm{mg} / \mathrm{l})\end{array}$ & $\begin{array}{c}\mathrm{Cl}^{-} \\
(\mathrm{mg} / \mathrm{l})\end{array}$ \\
\hline P1 & 7,52 & 180 & 107 & 100 & 28 & 7 & 5,5 & 130 & 5,16 & 1,43 & 2,05 \\
P2 & 7,58 & 182 & 108 & 100 & 27 & 8 & 5,5 & 132 & 3,87 & 1,59 & 1,40 \\
P4 & 6,72 & 156 & 87 & 82 & 23 & 6 & 4,5 & 106 & 5,56 & 3,20 & 9,92 \\
P5 & 7,38 & 291 & 157 & 150 & 42 & 11 & 4,5 & 191 & 6,54 & 0,81 & 1,12 \\
P7 & 6,30 & 98 & 52 & 46 & 11 & 4 & 5,5 & 63 & 5,22 & 2,18 & 1,78 \\
P11 & 6,84 & 177 & 100 & 96 & 25 & 8 & 6,5 & 122 & 3,57 & 2,16 & 3,51 \\
P23 & 6,89 & 188 & 111 & 100 & 20 & 12 & 7,0 & 135 & 5,29 & 0,81 & 2,44 \\
P24 & 7,24 & 245 & 127 & 122 & 39 & 6 & 5,0 & 155 & 7,33 & 1,43 & 3,09 \\
R30 & 8,05 & 139 & 78 & 76 & 23 & 5 & 5,0 & 95 & 6,26 & 0,81 & 1,35 \\
R31 & 7,61 & 125 & 70 & 70 & 20 & 5 & 4,5 & 85 & 6,83 & 0,81 & 1,04 \\
R32 & 8,18 & 125 & 72 & 70 & 20 & 5 & 4,0 & 88 & 5,17 & 0,81 & 0,75 \\
R33 & 8,20 & 124 & 70 & 66 & 19 & 5 & 4,5 & 85 & 5,82 & 0,81 & 2,15 \\
P13 & 4,95 & 27 & 4 & 6 & 1 & 1 & 2,0 & 5 & 1,79 & 4,94 & 2,91 \\
P16 & 5,26 & 20 & 4 & 8 & 0 & 2 & 1,0 & 5 & 1,28 & 2,69 & 1,99 \\
P17 & 5,62 & 20 & 6 & 8 & 1 & 1 & 1,0 & 7 & 1,28 & 0,81 & 2,12 \\
P25 & 5,18 & 40 & 3 & 10 & 1 & 2 & 3,5 & 4 & 1,28 & 8,03 & 4,89 \\
\hline
\end{tabular}

Las muestras presentan concentraciones de potasio muy bajas por lo que el límite de detección es de $1 \mathrm{mg} / 1$

(P: pozo; R: río)

De acuerdo con el diagrama de Piper (Fig. 7), se determinan dos grupos predominantes de aguas: una de tipo bicarbonatada cálcica y otra de tipo bicarbonatada clorurada sódica magnésica. Esta última corresponde con las muestras tomadas en los pozos perforados en la Unidad de Depósitos Aluviales Antiguos.

Este mismo comportamiento se logra observar con los diagramas de Stiff, los cuales se representan en la figura 8. En dicha figura se demuestran las variaciones en la forma de los diagramas, de acuerdo con las muestras analizadas. En la Unidad de Depósitos Aluviales Recientes se denota una predominancia en los iones de calcio y bicarbonato, mientras que en la Unidad de Depósitos Aluviales Antiguos se presenta un comportamiento en la composición química más equilibrado, donde no se observa mayor influencia de alguno de los iones.

De forma más detallada y utilizando los resultados mostrados en el cuadro 2 y en los diagramas elaborados, se destacan tres grupos principales de datos:

Grupo 1: Corresponde con las muestras tomadas en los pozos de los depósitos aluviales recientes (P1, P2, P4, P5, P7, P11, P23 y P24). Este grupo se caracteriza por presentar valores de $\mathrm{pH}$ de 5,9 a 7,05 ; conductividades eléctricas que oscilan entre

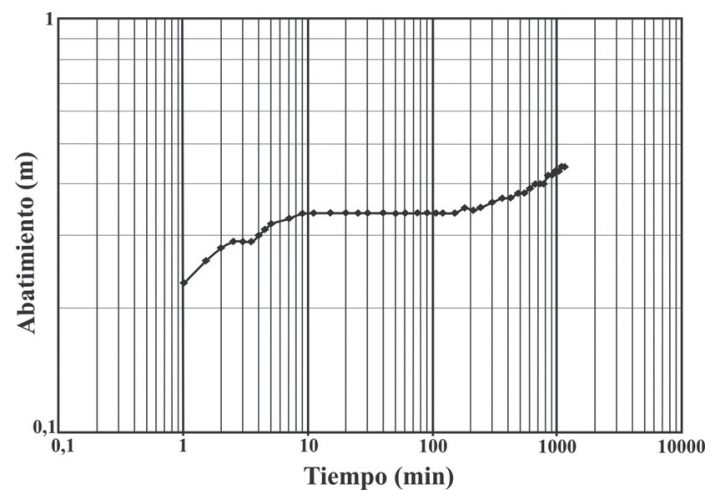

Fig. 6: Gráfico de la prueba de bombeo 


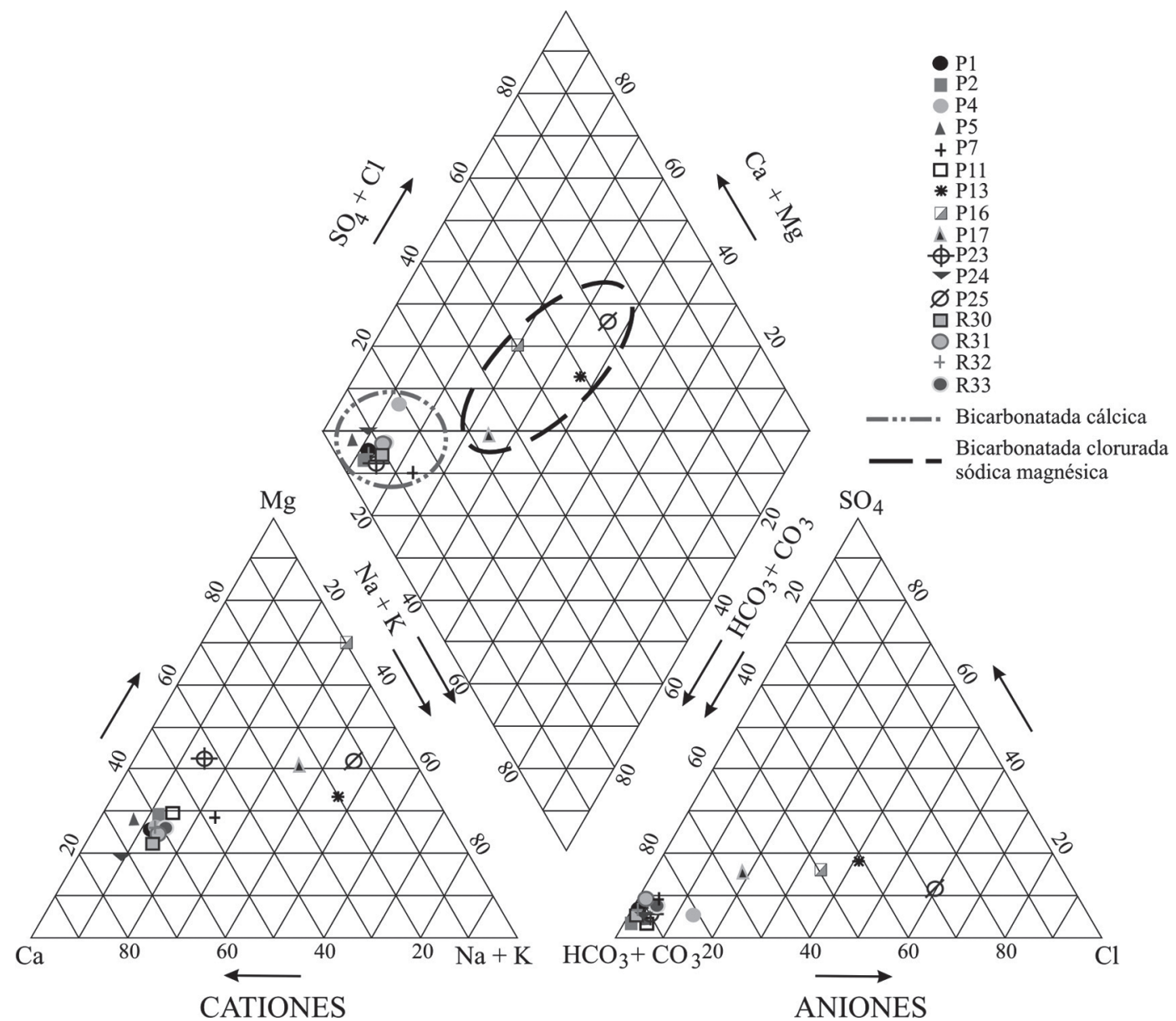

Fig. 7: Diagramas de Piper de las muestras de agua analizadas. Nótese la predominancia de dos grupos de muestras Grupo 1: P1, P2, P4, P5, P7, P11, P23, P24, R30, R31, R32 y R33; Grupo 2: P13, P16, P17 y P25

los 98 a los $291 \mu \mathrm{S} / \mathrm{cm}$. Los valores más altos de nitratos $(2,16 ; 2,18$; y $3,2 \mathrm{mg} / \mathrm{L})$ se encontraron en aquellos pozos donde no existe ninguna protección de la estructura del brocal y además se reconoció la existencia de contaminación bacteriológica por coliformes fecales del género Escherichia Coli, debido principalmente a la cercanía de tanques sépticos. Cabe destacar que además de la presencia de nitratos en la muestra $\mathrm{P} 7$, se reconoce que algunos parámetros analizados presentan un patrón inferior a las otras muestras de este grupo, lo cual podría indicar que el agua analizada pertenece al acuífero adyacente (Cuadro 3).

Grupo 2: Corresponde con las muestras tomadas en los pozos de los depósitos aluviales antiguos (P13, P16, P17 y P25). Este grupo presenta valores de $\mathrm{pH}$ ácidos de 4,43 a 5,14, así como bajas conductividades eléctricas que varían entre los 20 a los $40 \mu \mathrm{S} / \mathrm{cm}$. Se caracteriza por presentar los valores más bajos de iones mayores a excepción del cloruro y de nitratos, este último debido a la presencia de contaminación transmitida desde los tanques sépticos. Se encuentran valores de nitratos mayores que en el grupo 1, ya que la distancia de los tanques a los pozos muestreados es menor.

Tanto la alcalinidad como la concentración de bicarbonato son bajas debido al ambiente reductor en las que se encuentra estas aguas, por lo que es probable que la concentración de ácido carbónico sea predominante. Este ambiente ácido se origina posiblemente por la acumulación de materia orgánica dentro de la formación acuífera. 
Cuadro 3

Resultado de los análisis bacteriológicos medidos en el laboratorio

\begin{tabular}{cccc}
\hline Pozo & $\begin{array}{c}\text { Coliformes fecales } \\
(* 100 \mathrm{ml}-1)\end{array}$ & $\begin{array}{c}\text { E. coli } \\
\left(44,5^{\circ} \mathrm{C}\right)\end{array}$ & $\begin{array}{c}\text { Unidad de } \\
\text { Depósitos Aluviales }\end{array}$ \\
\hline P1 & Negativo & & \\
P2 & Negativo & 23 & \\
P4 & 240 & & \\
P5 & Negativo & 15 & \\
P7 & 15 & 43 & \\
P11 & 43 & & \\
P23 & Negativo & & \\
P24 & Negativo & & \\
P13 & Negativo & 43 & Antiguos \\
P16 & 93 & 240 & \\
P17 & 460 & & \\
P25 & Negativo & & \\
\hline
\end{tabular}

No se tomó muestras bacteriológicas en el río debido a la fragilidad del tipo de muestreo

Grupo 3: Corresponde con las muestras tomadas en el río Naranjo (R30, R31, R32 y R33). En este grupo se reconocen valores de $\mathrm{pH}$ de 7,75 a 8,37 y conductividades eléctricas de 124 a 139 $\mu \mathrm{S} / \mathrm{cm}$. Por lo general los rangos de los valores son consistentes, por lo que las características químicas se mantienen a lo largo del río. Los valores de nitratos son bajos debido a la dilución que se da con el río.

\section{CONCLUSIONES Y RECOMENDACIONES}

En el área de estudio se definieron siete unidades geológicas, de las cuales los depósitos aluviales tanto antiguos como recientes, corresponden las unidades hidrogeológicas más importantes.

El uso de la exploración geofísica representa una herramienta muy útil para la determinación de la geometría de los depósitos aluviales, así como precisar la profundidad del agua subterránea y variaciones de espesor del Acuífero Aluvial Naranjito, demostrando que la parte central de la cuenca se presenta como un sitio idóneo para realizar futuras perforaciones con fines de abastecimiento público.
Desde el punto de vista hidrogeoquímico se identificaron dos niveles de agua subterránea, almacenados en la Unidad de Depósitos Aluviales Recientes, el cual se clasifica de tipo bicarbonatada cálcica, y en la Unidad de Depósitos Aluviales Antiguos, clasificado como bicarbonatada clorurada sódica magnésica.

Aún considerando la existencia de una marcada diferencia topográfica, química y litológica entre ambos niveles, se determina a partir de las curvas isofreáticas una comunicación hidráulica lateral entre ambos acuíferos debido a sus características de confinamiento. La dirección de flujo en la parte superior de la cuenca es E - W, y en la parte media y baja es $\mathrm{S}-\mathrm{SW}$, siguiendo una trayectoria paralela al río Naranjo, donde en algunos tramos son efluentes, indicando una descarga del acuífero.

La similitud de los patrones químicos encontrados en las muestras de agua de los depósitos aluviales recientes y las muestras del río Naranjo, así como la determinación de los caudales diferenciales demuestran una interacción hidráulica entre el acuífero y el agua superficial, es por esto, que bajo este concepto de interacción entre los cuerpos de agua, es necesario tomar las medidas necesarias 


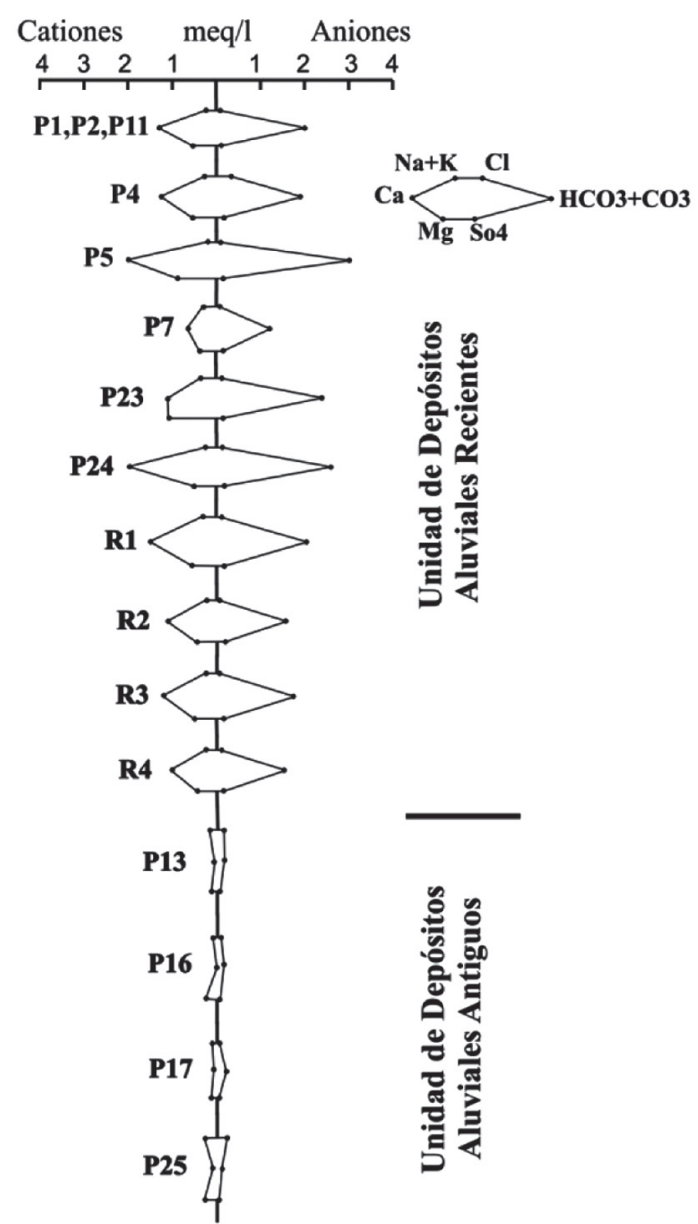

Fig. 8: Diagramas de Stiff para cada muestra de agua analizada. Nótese la diferencia entre las muestras tomadas en la Unidad de Depósitos Aluviales antiguos y los Depósitos Recientes y del río

en cuanto al control y manejo de la cuenca del río Naranjo, debido a la alta susceptibilidad a la contaminación del acuífero si las aguas del río se contaminaran, como por ejemplo el manejo, uso y control de agroquímicos utilizados en las plantaciones, así como derrames de hidrocarburos provenientes de las maquinarias utilizadas en la extracción de materiales del río.

Debido a la fuerte demanda de agua en la zona, es necesario evaluar la disponibilidad futura del recurso hídrico subterráneo y establecer planes de protección en las zonas de recarga y en las zonas de captura de pozos y manantiales, para así garantizar la calidad y potabilidad del agua. Además se requiere de la determinación de las zonas de mayor vulnerabilidad intrínseca así como la ubicación de los focos potenciales de contaminación por causa de un eventual cambio de uso del suelo.

\section{AGRADECIMIENTOS}

Esta publicación es un resultado parcial de la tesis de posgrado en Geología con énfasis en Manejo de Recursos Hídricos e Hidrogeología de la Universidad de Costa Rica, titulada "Evaluación de la vulnerabilidad intrínseca a partir de métodos geofísicos y determinación del potencial hídrico en el acuífero aluvial Naranjito, Quepos, Puntarenas", al comité evaluador M.Sc. Federico Arellano, M.Sc. Ingrid Vargas y M.Sc. Elena Badilla por todos los consejos y observaciones, al M.Sc. Mario Arias por sus sugerencias y comentarios, al Instituto Costarricense de Acueductos y Alcantarillados y al proyecto CARA por el financiamiento parcial de esta investigación.

\section{REFERENCIAS}

ACUÑA, J., 1975: Geología de la hoja Quepos.31 págs. Univ. de Costa Rica, San José [Inf. Campaña Geol.].

ARIAS, O., 2000: Geología y petrología del bloque Herradura (Cretácico Superior - Eoceno, Costa Rica).- 186 págs. L’ Université de Lausanne, Lausanne [Tesis Ph.D.].

ASTM., 2004: Standard guide for establishing nomenclature of ground-water aquifers.- 17 págs. ASTM Intern.

AZÉMA, J., GLAÇON, G., TOURNON, J. \& VILA, J., 1979: Precisiones acerca del Paleoceno de Puerto Quepos - Manuel Antonio y sus alrededores, provincia de Puntarenas, Costa Rica.- Inf. Sem. IGN, (2): 77-88. 
BAUMGARTNER, P., MORA, C.R., BUTTERLIN, J., SIGAL, J., GLAÇON, G., AZÉMA, J. \& BOURGOIS, J., 1984: Sedimentación y paleogeografía del Cretácico y Cenozoico del litoral pacífico de Costa Rica.- Rev. Geol. Amér. Central, 1: $57-136$.

BERGOEING, J.P. \& JIMÉNEZ, R., 1978: Investigaciones geográficas en el sector de Puerto Quepos - Manuel Antonio, provincia de Puntarenas, Costa Rica.- Inf. Sem. IGN, (1): 29-37.

CUSTODIO, E. \& LLAMAS, M., 1983: Hidrología Subterránea.- 2100 págs. Segunda edición. Tomo I y II. Ediciones Omega S.A., Barcelona.

DENYER, P., MONTERO, W. \& ALVARADO, G., 2003: Atlas tectónico de Costa Rica.-81 págs. Ed. UCR, San José.

DRAKE, P.G., 1989: Quaternary geology and tectonic geomorphology of the coastal piedmont range, Puerto Quepos area, Costa Rica.- 183 págs. The Univ. of New Mexico Albuquerque, New Mexico [Tesis M.Sc.].

FREEZE, R.A. \& CHERRY, J.A., 1979: Groundwater.- 604 págs. Ed. Prentice Hall, New Jersey.

GRANADOS, R. \& AGUILAR, T., 1983: Geopaleontología de la cuenca baja del río Savegre, Costa Rica.- Brenesia, 21: 229-260.

HENNINGSEN, D., 1965: La Fila Costeña del Pacífico de Costa Rica y su posición dentro del sistema montañoso centroamericano meridional.- 90 págs. Dir. Gral. Geol. Min. Petrol., Ministerio de Industria y Comercio, Costa Rica.

KRUSEMAN, G.P. \& DE RIDDER, N.A., 1994: Analysis and evaluation of pumping test data.- 377 págs. Inter. Inst. Land Reclamation and Improvement, The Netherland.

MALAVASSI, E., 1961: Some Costa Rican larger foraminiferal localities.- J. Paleont. 35: 498-501.

MORA, C.S., 1979: Proyecto hidroeléctrico Boruca. Informe de progreso $\mathrm{N}^{0} 5$, vol. I: Estudio geológico regional.- 193 págs. Inst. Costarricense de Electricidad, San José, Costa Rica.

SCHMIDT-EFFING, R., 1979: Alter und genese des Nicoya - Komplexes, elner ozeanischen paläokruste (Oberjura bis Eozän) im südlichen Zentralamerika.- Geol. Rdsch. 68: 457-494.

SENARA., 2003: Clasificación de zonas críticas de los recursos hídricos en la zona Parrita, Quepos y Dominical.- 65 págs. [Inf. int. SENARA].

TELFORD, W.M., GELDART, L.P. \& SHERIFF, R.E., 1996: Applied Geophysics [2 $2^{\mathrm{a}}$ ed.].770 págs. Cambridge University Press, Cambridge.

VALERÍN, E., 1982: Geología del área de Puerto Escondido y alrededores, Quepos, cantón de Aguirre, provincia de Puntarenas, Costa Rica.- 32 págs. Univ. de Costa Rica, San José [Inf. Campaña Geol.]. 